\title{
The utilisation of one district hospital emergency department by people with Parkinson's disease
}

\author{
Marion Harris ${ }^{\mathrm{a}}$, Margaret Fry ${ }^{\mathrm{b}, *}$ \\ a Emergency Department, Hornsby Hospital, Australia \\ b Northern Sydney Local Health District, Faculty of Health, University of Technology Sydney, Australia
}

\section{A R T I C L E I N F O}

\section{Article history:}

Received 4 August 2016

Received in revised form 8 November 2016 Accepted 15 December 2016

\section{Keywords:}

Emergency service

Parkinson disease

Neurodegnenerative diseases

Geriatrics

\begin{abstract}
A B S T R A C T
Background: Parkinson's disease (PD) is the second most common neurodegenerative disorder in Australia and the economic burden is more than $\$ 8.3$ billion a year and predicted to escalate. However, little is known of the trends and characteristics of people with Parkinson's disease presenting to emergency departments (ED).

Method: The study design was a 12 month retrospective medical record audit. The study was conducted in one metropolitan 300 bed district hospital in an outer suburb of Sydney.

Results: One hundred and twenty nine adult patients $(0.4 \%)$ with PD presented with a mean age of 79.5 years (SD 7.7); all were over 60 years of age. Of the 129 PD patient cases, there were 260 separate ED episodes of care with the majority $(n=151 ; 58.1 \%)$ of patients living in the community and presenting with falls $(\mathrm{n}=108 ; 41.5 \%)$. There was no statistical difference in triage code allocation when comparing PD patients and ED adult patients (over 60years). There was no difference in representation rate when comparing those over 80years $(n=128)$ with those under 80 years $(n=132)$. The average length of stay for PD patient episodes was $323 \mathrm{~min}$ (SD 225) compared with $193 \mathrm{~min}$ (SD 136) for ED patients. Younger adult patient episodes $(n=132 ; 51.0 \%)$ were admitted to hospital more frequently than those aged over 80 years (Pearson's X2 test $162.2 ; \mathrm{df} 1 ; \mathrm{p}<0.001$.

Conclusion: We identified in this study that people with PD have a high rate of falls, longer length of stay, high admission rate and represent frequently to the ED. Improved detection, management and a multidisciplinary approach for people with PD along with strict medication regime adherence is likely to improve safety, quality of life, reduce symptom aggravation and ongoing risk of falls.
\end{abstract}

(c) 2016 College of Emergency Nursing Australasia. Published by Elsevier Ltd. All rights reserved.

\section{Introduction}

The national economic burden of Parkinson's disease (PD) in the United States of America (USA) is estimated to exceed US\$14.4 billion in 2010 [1]. A global estimate of the number of people with Parkinson's disease (PD) over the age of 50 years is between 4.1 and 4.6 million [2]. In those 80 years and older PD has a prevalence of $2 \%$ [3]. By 2030, this number is expected to double to between 8.7 and 9.3 million people. In Australia 30 people are diagnosed each day with PD [4]. To date the number of Australians with PD is estimated at 80,000 and similarly is predicted to treble to 240,000 by 2033 [5].

$\mathrm{PD}$ is the second most common neurodegenerative disorder after Alzheimer's disease. The risk of developing PD increases

\footnotetext{
* Corresponding author.

E-mail addresses: Marion.Harris@health.nsw.gov.au (M. Harris), Margaret.Fry@uts.edu.au (M. Fry).
}

with age ${ }^{3}$ and is characterised by a progressive degeneration of pigmented neurons in the substantia nigra in the mid brain [6]. This causes a deficiency in dopamine, which is responsible for controlled and smooth muscle movement. It is a complex condition with diverse clinical features, neuropsychiatric symptoms and non-motor manifestations, which include autonomic dysfunction, gastrointestinal dysfunction and pain [7]. The cardinal motor features of Parkinson's include, resting tremor, bradykinesia, rigidity and postural instability with the latter occurring later in the disease. A PD diagnosis is made when two of the three cardinal signs, bradykinesia, rigidity and tremor, are evident. The goal of PD treatment is to minimise symptoms and manage medicines side effects.

As a result, for patients who attend specialty clinics where their Parkinson's is managed by a specialist physician or nurse, the key focus is to, maintain independence, maximise medicine compliance and treat medication side effects [8,9]. However, the Emergency Department (ED) presentation is frequently managed by a nonspecialist in PD, which may potentially result in suboptimal care 
as their condition can go undetected and unmanaged [6,9,10]. Evidence $[9,11,12]$ suggests that a lack of specialist knowledge and adherence to PD medication regimes can result in worsening clinical outcomes, such as motor symptoms, prolonged recovery, falls, and morbidity. The delay in establishing the patients home medication regime and administering their medications on time places the PD patient at higher risk of adverse events. These adverse events can lead to increased hospital length of stay, representations rates and augmented costs $[9,11,13-15]$.

Over 7.2 million attendances are managed by Australian EDs every year [16]. However, people with PD are a unique older person diagnostic group that present to EDs globally and are dependent on the clinician's assessment and recognition of the complexity of disease management. People with PD who present to the ED will usually have multiple co-morbidities [15] and so prompt identification, and timely and appropriate delivery of care is critical if optimal symptom control is to be achieved.

To date little is known or understood of the Australian ED trends and characteristics of people with PD. Therefore, the aim of this study was to describe the trends and characteristics of older people (60 years and over) with PD presenting to ED.

\section{Methods}

The study design was a 12 month retrospective medical record audit.

\section{Site}

The study was conducted in one metropolitan 300 bed district hospital in an outer suburb of Sydney, Australia, and the ED is a 22 bed referral centre.

\section{Sample}

A purposive sample of people with PD was enrolled in the study. Inclusion criteria required a diagnosis of $\mathrm{PD}$ (diagnosis determined by a neurologist and/or geriatrician) identified in the electronic medical record; 60 years of age or older; and living either in the community or in an aged care facility.

\section{Medical record audit}

A retrospective medical record audit was conducted between January 1 st 2014 to December 31st 2014 of people with PD. Patients were identified using the emergency department computer software program (FirstNet ${ }^{\mathrm{TM}}$ ). FirstNet is an electronic medical record information system that is a repository for all ED patient clinical encounters. At the study site, the ED has embedded a PD icon in Firstnet. Clinicians activate the PD icon for all relevant presentations. Data collected includes patient demographics (age, gender) and ED clinical information (presenting information, time and mode of arrival to the ED, triage code, doctor seen by time, treating doctor, discharge diagnosis and disposition). The ED database was then temporarily cross matched with the Aged Care Services in Emergency Team database. To maximise identification of PD patients the ED data set was cross matched to the ASET database which comprises all PD patients assessed within the ED model.

The New South Wales Ministry of Health initiated a new model of care-Aged Care Services in Emergency Team (ASET) that specialises in the early assessment and treatment of older persons with complex care needs presenting to ED. The ED model of care is supported by aged care medical and nursing specialists who routinely review all patients over 70 years of age or any patient that has a complex aged care need. As part of the ASET model a service database was established that captured: patient clinical characteristics (age, gender) and clinical information (presenting ED information, time of arrival to the ED, discharge diagnosis and disposition).

\section{Data analysis}

Quantitative data were analysed using IBM SPSS v21 ${ }^{\mathrm{TM}}$. Descriptive statistics (frequency and percentages) were used to summarise the study. Presenting ED diagnoses were grouped into categories. Categories were identified and grouped according to the ED symptom code in Firstnet based on SNOWMED [17]. Descriptive statistics were used to summarise and describe the characteristics of the sample (e.g. age, gender, time of arrival to the ED, triage code, seen by time, diagnosis and disposition from the ED). For normally distributed data, mean and standard deviations are presented. Age was considered a categorical variable. Comparison of groups was done using the Pearson Chi-squared test (gender age and triage category). For the study, statistical significance was set at $\mathrm{p}<0.05$.

\section{Ethics approval}

Ethical approval to undertake the study was obtained from the Human Research Ethics Committee of the Local Health District (HREC/LNR/15/HAWKE/252). Access to data was approved to analyse patient medical records and the researchers operated in accordance with the Australian National Health and Medical Research Council guidelines [18]. The ethical conduct of research was maintained during and after the research and data sources were stored in password protected files. To maintain confidentiality and privacy, patients were de-identified and re-coded on data collection. The study was conducted in accordance with the approved protocol and is reported using STROBE guidelines [19].

\section{Results}

In 2014, this ED had 37341 patient presentations which consisted of $11122(29.7 \%)$ paediatric and 26219 (70.2\%) adult patients. Of the adult population 10490 (28.1\%) patients were over the age of 60 years. Patient demographics are described in Table 1 . Of the 129 PD patient cases there were 260 separate ED episodes of care with the majority arriving by ambulance. The 260 episodes of care comprised 139 males (53.5\%) and 121 females (46.5\%). There was a statistical difference with more male presentations (Pearson's $X^{2}$ test 4.40; df $1 ; \mathrm{p}<0.05)$.

The mean time to be seen by ED staff for PD patients was $23 \mathrm{~min}$ (SD 26). For those patients arriving between business hours ( 800 and $1700 \mathrm{~h}$ ), the mean time to be examined was $20 \mathrm{~min}$ (SD 21) compared to after hours, which was $30 \mathrm{~min}$ (SD 32). Most PD patients visited the ED on a weekday ( $n=192 ; 73.9 \%)$. For the episodes of care most were classified as non-urgent and allocated a triage category $4(n=163,62.8 \%)$ or $5(n=20 ; 7.7 \%)$ (Table 1$)$. There was no statistical difference in triage code allocation when comparing PD patients and ED adult patients (60 years and over) (Pearson's $X 2$ test 3.938 df $1 \mathrm{p}<0.05$ ).

The most common ED Triage symptom presentation was falls $(\mathrm{n}=118 ; 45.4 \%$ ) (Table 2). Of the 260 episodes of ED care there was no difference in representation rate when comparing age groups, younger than 79 years with those 80 years and over. For all episodes of care there were more presentations originating from the community $(n=152 ; 58.5 \%)$ than aged care facilities $(n=108 ; 41.5 \%)$ for either age group (Table 2 ).

There was wide variation in length of stay when comparing aged care facilities and community dwelling residents. Patients from an aged care facility had a longer arrival to discharge time on weekends 
Table 1

Characteristics of PD Patients ( $\mathrm{N}=129)$.

\begin{tabular}{|c|c|c|c|c|}
\hline & & $\mathrm{N}(\%)$ & & \\
\hline \multicolumn{5}{|l|}{ Gender } \\
\hline Male & 69 & $(53.5 \%)$ & & \\
\hline Female & 60 & $(47.5 \%)$ & & \\
\hline Total & 129 & & & \\
\hline Age (Mean \pm 1SD) & & $79.5(7.7)$ & & \\
\hline \multicolumn{5}{|c|}{ Number of re-presentation $(n=260)$} \\
\hline Male & & 139 & & \\
\hline Female & & 121 & & \\
\hline Single patient presentation & & 58 & & \\
\hline Two patient presentations & & 38 & & \\
\hline Three patient presentations & & 20 & & \\
\hline Four patient presentations & & 7 & & \\
\hline Five patient presentations & & 4 & & \\
\hline Seven patient presentations & & 1 & & \\
\hline Eleven patient presentations & & 1 & & \\
\hline \multicolumn{5}{|c|}{ Mode of arrival for re-presentations } \\
\hline Ambulance & & 217 & $(83.5 \%)$ & \\
\hline Private car & & 40 & $(15.4 \%)$ & \\
\hline Walking & & 3 & $(1.1 \%)$ & \\
\hline \multicolumn{5}{|c|}{ Triage category for re-presentations } \\
\hline Triage category 1 & & 2 & $(0.8 \%)$ & \\
\hline Triage category 2 & & 19 & $(7.3 \%)$ & \\
\hline Triage category 3 & & 56 & $(21.5 \%)$ & \\
\hline Triage category 4 & & 163 & $(62.8 \%)$ & \\
\hline Triage category 5 & & 20 & $(7.7 \%)$ & \\
\hline Triage to "seen by" (minutes) & & Mean & SD & $\mathrm{N}$ \\
\hline 800am-500pm (mean SD) & & 20 & 21 & 148 \\
\hline Out of hours(mean SD) & & 30 & 32 & 112 \\
\hline mean & & 23 & 26 & 260 \\
\hline Average length of stay (min) & & Mean & SD & $\mathrm{N}$ \\
\hline PD patients & & 323 & 225 & 260 \\
\hline All ED Patients & & 193 & 136 & \\
\hline \multicolumn{5}{|l|}{ Arrival by day } \\
\hline Weekday & & 192 & $(73.9 \%)$ & \\
\hline Weekend & & 68 & $(26.1 \%)$ & \\
\hline Monday & & 36 & $(13.8 \%)$ & \\
\hline Tuesday & & 44 & $(16.9 \%)$ & \\
\hline Wednesday & & 49 & $(18.8 \%)$ & \\
\hline Thursday & & 28 & $(10.6 \%)$ & \\
\hline Friday & & 35 & $(13.5 \%)$ & \\
\hline Saturday & & 36 & $(13.8 \%)$ & \\
\hline Sunday & & 32 & $(12.3 \%)$ & \\
\hline \multicolumn{5}{|l|}{ Arrival time of day } \\
\hline $8: 00-500 p m$ & & 148 & $(56.9 \%)$ & \\
\hline Out of hours & & 112 & $(43.1 \%)$ & \\
\hline Average length of stay (min) & & Mean & $\mathrm{SD}$ & \\
\hline PD patients & & 323 & 225 & \\
\hline All ED Patients & & 193 & 136 & \\
\hline \multicolumn{5}{|l|}{ Disposition } \\
\hline Admitted & & 162 & $(62.3 \%)$ & \\
\hline Discharged home & & 97 & $(37.3 \%)$ & \\
\hline Left at own risk & & 1 & $(0.4 \%)$ & \\
\hline
\end{tabular}

Table 2

Presenting triage symptoms for ED Visit.

\begin{tabular}{|c|c|c|c|}
\hline & Community & Aged care Facility & Total \\
\hline \multicolumn{4}{|l|}{ Presenting symptom } \\
\hline Falls & $68(26.1 \%)$ & $50(19.2 \%)$ & $118(45.4 \%)$ \\
\hline Pain & $24(9.2 \%)$ & $9(3.4 \%)$ & $33(12.7 \%)$ \\
\hline Infection & $15(5.7 \%)$ & $14(5.3 \%)$ & $29(11.2 \%)$ \\
\hline Review & $7(2.6 \%)$ & $9(3.4 \%)$ & $16(6.2 \%)$ \\
\hline Mental health disorder & $10(3.8 \%)$ & $4(1.5 \%)$ & $14(5.4 \%)$ \\
\hline Neurology & $7(2.6 \%)$ & $5(1.9 \%)$ & $12(4.6 \%)$ \\
\hline Respiratory disorder & $4(1.5 \%)$ & $7(2.6 \%)$ & $11(4.2 \%)$ \\
\hline Others & $3(1.1 \%)$ & $7(2.6 \%)$ & $10(3.8 \%)$ \\
\hline Adverse drug events & $6(2.3 \%)$ & $2(0.7 \%)$ & $8(3.1 \%)$ \\
\hline Gastroenterology & $5(1.9 \%)$ & $1(0.3 \%)$ & $6(2.3 \%)$ \\
\hline Cardiac & $3(1.1 \%)$ & $0(0.0 \%)$ & $3(1.2 \%)$ \\
\hline Total & 152 & 108 & 260 \\
\hline
\end{tabular}

between $5 \mathrm{pm}$ and $8 \mathrm{am}$ (620 min) compared with patients from the community ( $359 \mathrm{~min}$ ) for the same period.

The average length of stay for PD patient episodes was $323 \mathrm{~min}$ (SD 225) compared with $193 \mathrm{~min}$ (SD 136) for ED adult patients (60 years and over). The majority of episodes required the patient to be admitted ( $\mathrm{n}=162 ; 62.0 \%)$.

Adult patients younger than 80 years $(n=132 ; 51.0 \%)$ were admitted to hospital more frequently than those aged over 80 years (Pearson's $X^{2}$ test 162.2 ; df 1 ; $\left.p=0.001\right)$. There were $98(38.0 \%)$ episodes for which the patient was discharge home or back to an aged care facility. When comparing disposition for those aged over 80 years the majority were discharged $(n=98 ; 37.7 \%)$ back to community $(n=48 ; 49.0 \%$ ) or an aged care facility $(n=50 ; 51.0 \%)$. For those aged over 80 years they were more likely to be discharged back to an aged care facility $(n=97 ; 37.3 \%)$.

\section{Discussion}

This is the first Australian study that has examined the trends and characteristics of people with PD presenting to an Australian ED. This trend data has demonstrated that people with PD have a high falls injury rate, admission rate, longer ED length of stay, and multiple presentations. EDs need to consider their role beyond episodic care as increasingly vulnerable ageing population groups are presenting repeatedly.

This study identified that of the 129 patients, there were 260 presentations over 12 month period and that 33 patients presented more than three times to the ED. This suggests greater preventative strategies need to be considered especially given that the majority of these patients lived in their own home. Further, early involvement of family and carers and stronger links with specialist clinics as well as primary care, may provide for more optimal safety discharge strategies in the ED [20-22].

This study further supports the evidence that people with Parkinson disease frequently present to an ED with injuries as a result of falls [15]. For people with PD episodes of falls are the result of progressive postural instability, autonomic dysfunction, orthostatic hypotension, and mental health changes that may result in the need for management, diagnostic interventions and hospital admission [15]. Falls are associated with significant adverse events, which can include injury, disability or death [23]. One patient in the study was admitted 11 times for falls and regularly suffered minor head injuries. Falls can be a symptom of PD progression and may also relate to confusion, delirium and/or dementia [8]. Interventions need to be developed to reduce both risk and fear of falling to maximise independence, quality of life and reduce ED representations.

ED presentations and hospitalisations related to falls remain a significant issue for patients with PD [22]. The cost implications to the health sector are extensive as once hospitalised, people with PD are at a greater risk for hospital morbidity and mortality [1,2,14]. The Australian Commission on Safety and Quality in Health Care has developed a National Safety and Quality Health Service Standards [24]. This document, for use in hospitals, residential aged and community care, presents ten Service Standards of which Standard Ten relates to 'Preventing Falls and Harm from Falls'. These guidelines are evidence based and a valuable resource that can be used by ED clinicians when assessing the needs of people with PD [24]. However, the standards do not recommend the use of a national screening tool to assess severity or falls risk for patients. Further research is needed to determine and implement the most appropriate falls screening tool as this may assist with determining appropriate interventions and/or reducing ED representations.

A range of intervention fall strategies have been outlined in a recent systematic review [23] for older adults. For example, a reduc- 
tion in falls was identified in older persons who received Vitamin $D$ supplementation, visual acuity assessments and exercise and muscle strengthening encouragement. A comprehensive ED assessment that includes these elements may improve detection and therefore ameliorate the risk of falls in this group. Further, there is evidence that lack of falls awareness and appropriate patient/carer knowledge, competing risks, and patient compliance can also influence fall rates [23]. As a result a range of fall intervention strategies may be needed in EDs to better target these dimensions.

Routine screening procedures for cognitive impairment, in the $\mathrm{ED}$, may assist in the early recognition of deterioration in PD patients [3,25]. Australian EDs manage 1.4 million people aged 60 years and over every year [26]. Of those 60 years and over approximately $26 \%$ will have a cognitive impairment [26,27]. In this population, poor medication adherence can give rise to confusion, delirium and agitation, and result in family/carer distress.

The findings of this study highlighted that many people with PD live in the community. To ensure people with PD remain in their own homes independently and safely, ED discharge practices need to be reviewed. However, ED discharge interventions may need to vary depending on disease progression. There is evidence to suggest that a more comprehensive and multidisciplinary approach can lead to safer ED discharge [28-30]. Further, the early involvement of pharmacists and allied health professionals in conjunction with the treating team and nursing staff can facilitate a more personalised care plan [31]. Investigation is needed into other quality of life dimensions, such as those provided by allied health, which may assist to reduce admission and representation rates in this group. To this end, the development of a clinical pathway may assist to improve care and discharge consistency and strengthen multidisciplinary input into the management of PD patients in the ED.

While PD patients are in the ED their reliance on their medication regime to minimise symptoms remains the same as for those in the community. However, EDs traditionally may not stock the required medicines for $\mathrm{PD}$. As a result, subsequent medication delay may be experienced by people with $\mathrm{PD}$, which can jeopardise finely tuned symptom control. The result of non-adherence to medication regimes in the ED can further potentiate risk of falls, which can impact on hospital costs, length of stay and adverse event rates. Therefore, these time critical medicines need to be readily available to ensure prompt and appropriate administration. ED processes need to be implemented to ensure ready access to PD medicines twenty-four hours per day for this vulnerable group.

Mental Health conditions are common in people with PD (30-40\%) [4,32]. While there were a small number of patients within the study with mental health conditions, there was no evidence of referral to mental health services. However, within the literature there is mounting evidence that PD patients will often experiences depression and anxiety [8,32]. Further consideration needs to be given to appropriate screening and/or referral to mental health services during ED stay or discharge. Therefore, clinicians need to screen for these conditions to ensure independence, quality of life and minimal impact on physical symptoms [4].

This study supports the evidence that people with PD generally have a longer hospital length of stay compared to other patient groups $[11,33]$. However, with disease progression there is also an increased incidence in cognitive and psychiatric impairment. Consequently, people with PD and dementia are usually hospitalised twice as long compared to non-dementia PD patients [32]. For PD patients with dementia, particular caution and frequent reassessment by a multidisciplinary team is needed to minimise hospital stay and enhance quality of life. With the global prediction of an increase in PD prevalence the longer length of stay in this patient group may pose a future challenge in ED.
To improve the care of older persons presenting to the ED the New South Wales (NSW) Ministry of Health implemented specialised Aged Care Service teams [34] and is known as Aged Care Services in Emergency Team (ASET) [35]. However, like many new health care models that are widely introduced they often have limited funding, which reduces service cover. Not all ASET teams work seven days a week and some are limited to business hours only. As a result delays may be experienced on weekends and after hours. However in this study site the service covers fourteen hours a day seven days a week. New models of care need to profile the targeted service to better match resources to workload demands.

Given the prevalence and expected increase of PD globally, ED education and orientation programs need to include knowledge and care practices specific to the PD patient [11]. Further, evidence based clinical guidelines will assist in the early detection and management of PD presentations [36,37]. Greater collaborative care with allied health, such as pharmacists, to assist with improving medication adherence regimes will avert potential adverse events. When considering ED pharmacy medicine stock, as for other patient conditions, the availability of PD medicines in the ED would ensure timely administration of medications. When a PD presentation is recognised early documentation of medicines regimes and referral to allied health may reduce adverse outcomes and improve emergency care $[3,38]$.

Further research is needed to explore the specific management of people with PD in ED and during hospitalization. There is also a need to explore the quality of care and what is optimal care in the ED. To better support ED discharge processes and safety, different PD models of care based within the community need to be tested [21]. Early warning systems, triggers, need to be developed in ED, to alert services to the presentation of a person with PD. In addition, educational input from specialists in PD need to be incorporated into the ED and inpatient ward education programs to improve management and discharge processes to prevent unnecessary representations and harm.

\section{Limitations}

There are a number of key aspects that should be kept in mind when giving consideration to the results of this study. While significant resources were in place to identify people with PD there may be cases which have been missed due to workload and ASET team availability. Further, ED patients that did not wait for treatment or left at own risk may have included PD cases. Patients may have presented to the ED with undetected and or undiagnosed PD and as a result may have been missed. Further communication barriers with patients or aged care facilities may have led to cases being missed in the database. The trends and incidence rate found in the study may not be representative of larger tertiary referral EDs.

\section{Conclusion}

This study has identified that people with PD have a high fall rate, longer length of stay, high admission rate and frequently represent to ED. Better detection and management of people with PD on arriving to the $\mathrm{ED}$, along with medication regime adherence, is likely to reduce symptom aggravation and ongoing falls risk.

There is evidence to suggest that early multidisciplinary referral and discharge screening for this vulnerable patient group may improve quality of life and safety by reducing serious injuries from falls. Further, clinical practice guidelines may assist to strengthen multidisciplinary engagement, consistency and improve the quality of ED care for people with PD. 


\section{Conflict of interest}

Author Margaret Fry is a Deputy Editor of the Australasian Emergency Nursing Journal but had no role in the peer review or editorial decision-making of this manuscript whatsoever.

\section{Provenance}

MH and MF conceived the study, design the trial and obtained the research funding. MH and MF oversaw the participating site and managed the quality of data collection. MF provided statistical advice on the study design, sample and analysis of the data. $\mathrm{MH}$ and MF were involved in the drafting of this manuscript. MF takes responsibility for the paper as a whole.

\section{Funding}

No funding was obtained for this study or the writing of the manuscript.

\section{References}

[1] Kowal SL, Dall TM, Chakrabarti R, Storm MV, Jain A. The current and projected economic burden of Parkinson's disease in the United States. Mov Disord $2013 ; 28$.

[2] Dorsey E, Constantinescu R, Thompson J, et al. Projected number of people with Parkinson disease in most populous nations, 2005 through 2030. Neurology 2007;68:384-6.

[3] Ghosh R, Liddle BJ. Emergency presentations of Parkinson's disease: early recognition and tretment are crucial for optimum outcome. Postgrad Med 2011;87:125-31.

[4] Beyond Blue, Parkinson's Australia: Parkinson's disease, anxiety and depression. vol 2016, 2016.

[5] Parkinson's Australia: The productivity commission inquiry into aged care services. Canberra,ACT : Parkinson's Australia, 2010; 1-21.

[6] Jones SL, Hindle J. Parkinson's disease in the acute hospital. Clin Med $2011 ; 11: 84-8$.

[7] Stacy M, Davis TL, Heath S, et al. The clinicians' and nurses' guide to Parkinson's disease. Medscape 2009 weblink www.medscape.org/viewarticle/701955.

[8] Levine C, Fahrbach K, Sideowf A, Estok R, Ludensky V, Ross S. Diagnosis and Treatment of Parkinons's Disease: A Systematic Review of the Literature. Evidence/Report/Technology Assessment Number 57. Rockville MD: Agency for Healthcare Research and Quality; 2003.

[9] Guneysel O, Onultan O, Onur O. Parkinson's disease and the frequent reasons for emergency admission. Neuropsychiatr Dis Treat 2008;4:711-4.

[10] Lowthian J, Curtis A, Cameron PA, Stoelwinder J, Cooke M, McNeil JJ. Systematic review of trends in emergency department attendances: an Australian perspective. Emerg Med J 2011;28:373-7.

[11] Aminoff MJ, Christine CW, Friedman JH, et al. Management of the hospitalized patient with Parkinson's disease: current state of the field and need for guidelines. Parkinsonism Relat Disord 2011;17:139-45.

[12] Skudlarska BA, Argento V, Raccio D, et al. Mental status change and hallucinations in an elderly patient with Parkinson's disease as a result of proper medication supervision-the case for geriatric consultation in hospitalized frail elderly. Eur Geriatr Med 2011;25:S109

[13] Grubb E, Treglia M, Lage M, Castelli-Haley J. Comparison of hospitalizations, emergency room visits, fractures, and falls among parkinson disease patients who initiated therapy with selegiline or rasagiline: a retrospective study. Value Health 2013;16(3):A105.
[14] Hassan A, Wu SS, Schmidt P, et al. High rates and the risk factors for emergency room visits and hospitalization in Parkinson's disease. Parkinsonism Relat Disord 2013;19:949-54.

[15] Braga M, Pederzoli M, Antonini A, Beretta F, Crespi V. Reasons for hospitalization in Parkinson's disease: a case-control study. Parkinsonism Relat Disord 2014;20:488-92.

[16] Australian Institute of Health and Welfare. Australian Hospital Statistics 2013-14 Emergency Department Care Health Services Series No. 58. Cat. No. HSE 153. Canberra: Australian Institute of Health and Welfare; 2014.

[17] Dinh MM, Berendsen Russell S, Bein KJ, et al. Understanding drivers of demand for emergency service trends in years 2010-2014 in New South Wales: an initial overview of the DESTINY project. Emerg Med Australas 2016;28:179-86.

[18] National Health and Medical Research Council. Guidelines approved under section 95A of the Privacy Act. 2014: Australian Government; 1988

[19] Vandenbroucke JP, von Elm E, Altman DG, et al. Strengthening the reporting of observational studies in epidemiology (STROBE): explanation and elaboration. Int J Surg 2014;12:1500-24.

[20] Temlett J, Thompson P. Reasons for admission to hospital for Parkinson's disease. Intern Med J 2006;36:524-6.

[21] Jones B, Hopkins B, Wherry S, Lueck C, Chandi P, Dugdale P. Evaluation of a regional australian nurse-led Parkinson's service using the context, input, process and product evaluation model. Clin Nurse Specialist J 2016.

[22] Lubomski M, Rushworth RL, Tisch S. Hospitalisation and comorbidities in Parkinson's disease: a large Australian retrospective study. Neurol Neurosurg Psychiatry 2014;86:324-30.

[23] Michael Y, Lin J, Whitlock E, et al. Primary care relevant interventions to prevent falls in older adults: a systematic evidence review for the U.S. preventative services task force. Ann Emerg Med 2010;153:815-24.

[24] ACSQHC, National Safety and Quality Health Service Standards. In: Care ACoSaQiH, ed. Sydney : ACSQHC, 2012; 1-80.

[25] Carpenter CR, Platts-Mills TF. Evolving prehospital, emergency department, and inpatient management models for geriatric emergencies. Clin Geriatr Med 2013;29:31-47

[26] Gray LC, Peel NM, Costa AP, et al. Profiles of older patients in the emergency department: findings from the interRAI multinational emergency department study. Ann Emerg Med 2013;62:467-74.

[27] Fry M, Chenoweth L, Arendts G. Assessment and management of acute pain in the older person with cognitive impairment: a qualitative study. Int Emerg Nurs 2016;24:54-60

[28] Department of Health, Western Australia: Parkinson's Disease Services Model of Care. Perth, WA: Aged Care Network, Department of Health, Western Australia, 2008.

[29] American College of Emergency Physicians, The American Geriatrics Society, Emergency Nurses Association, The Society for Academic Emergency Medicine: Geriatric Emergency Department Guidelines, 2014.

[30] Alzheimer's Australia: Dementia Care in the Acute Hospital Setting. Sydney: Alzhiemers Australia, 2014.

[31] Parkinson's Australia: Parkinson's Australia Submission to National Health \& Hospitals Reform Commission. Australia: Parkinson's Australia, 2008 1-16.

[32] Oguh O, videnovic A. Inpatient management of parkinson disease. The Neurohospitalist 2012;2:28-35.

[33] Gerlach O, Broen M, van Domburg P, Vermeij A, Weber W. Deterioration of Parkinson's disease during hospitalization: survey of 684 patients. BMC Neurol 2012;12.

[34] NSW Health Integrated Care: NSW Aged Care Services in Emergency Teams Practice Guidelines. In: Care I, ed.: NSW Health, 2014.

[35] Clinical/Patient Services-Aged Care: NSW Aged Care Services in Emergency Teams Practice Guidelines. NSW Ministry Health 2014.

[36] Gerlach O, Winogrodzka A. Weber W. Clinical problems in the hospitalized Parkinson's diseae patient: systematic review. Mov Disord 2010;26:197-208.

[37] Hou J, Wu L, Moore S, et al. Assessment of appropriate medication administration for hospitalised patients with Parkinson's disease. Parkinsonism Relat Disord 2012;18:377-81.

[38] Derry C, Shah K, Caie L, Counsell C. Medication management in people with Parkinson's disease during surgical admissions. Postgrad Med J 2010:86:334-7. 